\title{
Epidemiology and Vaccine Efficacy of Delta Variant
}

\author{
Hassan Naji
}

ABSTRACT

\begin{abstract}
Several variants of concern (VOCs) of SARS-CoV-2 have emerged after its first outbreak in $\mathbf{2 0 2 0}$. These VOCs possess mutations in the spike protein that confers enhanced rate of infection and transmission of these VOCs. Among these VOCs, the delta/B.1.617.2 variant was identified in late 2020 in India and soon spread to several countries around the globe. Currently, it is the most widely sequenced variant among new infected individuals with SARS-CoV-2 infection. This variant is also found to be associated with COVID-19 infection in vaccinated individuals, but the severity of disease and rate of hospitalization are low among these individuals as compared to unvaccinated individuals. With the emergence of new variants of SARS-CoV-2 due to continuous mutations, it is suggested that vaccination plays a significant role in preventing the transmission of disease and disease severity.
\end{abstract}

Keywords: AstraZeneca, BNT162b2, coronavirus, COVID-19, Delta variant, Moderna and ChAdOx1, mRNA-1273, Pfizer-bioNTech, RNA load, SARS vaccine, SARS-CoV-2, several variants of concern (VOCs), spike protein.

\author{
Submitted : November 30, 2021 \\ Published : December 29, 2021 \\ ISSN: $2593-8339$ \\ DOI: $10.24018 /$ ejmed.2021.3.6.1162 \\ H. Naji* \\ Purdue University Northwest, United \\ States of America. \\ (e-mail: hnaji@pnw.edu) \\ *Corresponding Author
}

\section{INTRODUCTION}

Since its emergence, SARS-CoV-2 underwent various mutations and several variants of concerns (VOCs) emerged with increased infectivity and decreased vaccine effectiveness. The VOCs vary in the amino acid sequence of the spike protein and contain several sequential changes. These changes include alpha, beta, gamma, and the delta variants. This serial emergence of VOCs depicts the increased effective reproduction numbers that cause the VOCs to dominate already existing strains in the light of similar disease control interventions. These VOCs are related to higher risk of admission to hospitals, intensive care units and death, according to various predictive factors including age [1]. World Health Organization (WHO) revised the nomenclature for the B.1.617.2 variant of SARS-CoV-2 in April 2021 and named it as "Delta" [2]. By the end of June 2021, delta variant of SARS-CoV-2 became the most widely sequenced variant worldwide [3]. As of September 28, 2021, globally 187 countries and several other areas and territories reported individuals infected with delta variant of SARSCoV-2. Meantime, delta variant of SARS-CoV-2 is the predominant VOC among various populations even having access to vaccines [4]. The emerging delta variant has gained attention globally due to enhanced global public health risk and being the dominant variant in the largest outbreak of SARS-CoV-2 infection in India since March 2021 [5].

The delta variant of SARS-CoV-2 has high transmission rates as compared to other circulating variants. For delta variant, the window period from the exposure of virus to its detection in individuals is almost 3.7 days [5]. According to [6], vaccinated individuals can also spread delta variant of SARS-CoV-2. Several vaccine breakthrough SARS-CoV-2 infections are observed particularly due to selection pressures and genetic drift that result in the emergence of new VOCs. However, the disease spectrum is likely be milder with further implementation of vaccination programs [7]. This paper focuses on the emergence and epidemiology of the delta variant and vaccine effectiveness against the delta variant of SARS-CoV-2.

\section{EMERging OF DELTA VARIANT}

The delta variant first emerged in October 2020 in the Maharashtra state of India [8], then in December 2020, in Ontario, Canada with the mutations E484K and/or N501Y [9]. The sub-lineage B.1.617.1 with the spike protein mutation L452R was first detected in India, followed by the sub-lineage B.1.617.2/delta variant with the same mutation. This mutation was similar to that of B.1.429 lineage, known as California variant. Other mutations that were found in these sub-lineages include D950N, P681R, D614G, E484Q, T478K, $\triangle 157-158$ and T19R in the receptor-binding domain (RBD) of the spike protein. The mutations, E484Q, T478K and L452R are considered to be responsible for enhanced angiotensin-converting enzyme 2 (ACE2) receptors binding, and the P681R mutation in the furin cleavage domain results in increased S1-S2 cleavage rate associated with higher transmissibility of the delta variant [10]. As of May 19, 2021, the delta variant had been detected globally in 43 countries across 6 continents in GISAID [11].

In the U.S. in late June 2021, the average number of reported cases within 7 days rapidly increased to about 12,000 . On July 27 , this number further increased to 60,000 even with the widespread availability of vaccine. New data showed that the delta variant was responsible for the 
increased transmission rates, even in vaccinated persons, as compared to that of other variants, and was highly infectious [12]. Further transmission of delta variant occurred around the globe due to travel history of individuals.

\section{EPIDEMIOlOGY OF DELTA VARIANT}

After its emergence in late 2020, in Maharashtra, the delta variant spread throughout India. In mid-April 2021, the delta variant was the most frequently reported variant in the country. A total of 385 out of 604 SARS-CoV-2 genomic sequences in Delhi reported to GISAID were delta variant. On May 1, 2021, the SARS-CoV-2 infected cases reached more than 400,000 and 4000 deaths were reported each day in India. Studies suggest that the delta variant is more transmissible and has better capability to evade immune system response generated by prior infection as compared to that of previous circulating SARS-CoV-2 lineages. The transmission rate of delta variant as compared to that of other circulating lineages in Mumbai, India was estimated to be 1.1-1.4-fold higher and its ability to evade immunity was also found to be $20-55 \%$ higher [13].

The delta variant spread to the United Kingdom because of travel from India and community transmission [14]. In May 2021 , more than $75 \%$ cases of SARS-CoV-2 infection in England were caused by delta variant linked to travel history and community transmission among individuals [15].

The B.1.617/delta variant of SARS-CoV-2 emerged in Ontario, Canada from February-June 2021 after the emergence of VOCs namely, B1.1.17/alpha, B.1.351/beta and P.1/gamma variants with N501Y mutation. In late April 2021, all new infections in Ontario were attributed to these VOCs. According to a retrospective cohort study of 212,332 confirmed SARS-CoV-2 infected individuals, $77 \%$ individuals were reported to be infected with N501Y-positive VOC and $2.7 \%$ were reported as probable delta infections. This study also found $49 \%, 86 \%$ and $51 \%$ relative increased risk of hospitalization, intensive care unit (ICU) admission and death, respectively, in individuals infected with delta variant as compared to those infected with non-VOC and N501Y-positive strain, after adjustments for sex, age, comorbidities, vaccination status, temporal trend and health unit [2].

In China, the first infected individual with delta variant was identified in Guangzhou, Guangdong, on May 21, 2021. The delta variant soon resulted in an outbreak in Guangdong. Starting from this first index case to the last infected individual reported on June 18, 2021, a total of 167 individuals infected with the delta variant of SARS-CoV-2 were reported. A study of the quarantined individuals reported significantly higher viral loads of $\sim 1000$ times higher in delta variant infected COVID-19 patients on the first positive PCR test than in those infected with the clade 19A or 19B of SARS-CoV-2. It depicts higher virulence rate of delta variant during early phase of infection among asymptomatic individuals [5]. All the VOCs including the delta variant have also been reported in Canada, Africa, Brazil, the Cayman Islands, Barbuda and Antigua, Guatemala, Panama, Mexico, Sint Maarten, French Guiana, Uruguay, Argentina, Costa Rica, Chile, Guadeloupe, Suriname, Aruba, Curacao, Martinique, Puerto Rico, and the
United States of America by August 20, 2021 [16].

Currently, delta variant is the predominant variant among patients infected with SARS-CoV-2. According to the [4] and as of November 25, 2021, there is a total of 259,502,031 confirmed cases of COVID-19, including 5,183,003 deaths. The predominant SARS CoV-2 variant is the delta variant which accounts for more than $99 \%$ of the infection reported leading to an overwhelming increase in hospitalization and death rates. Delta variant is reported to be more than twice as contagious as previous variants. Unvaccinated individuals are more at risk of infection and are contributing to the highest spread of cases and severe outcomes [4].

According to the [17], individuals with preexisting conditions such as chronic lung diseases, cardiovascular diseases, chronic kidney diseases, immunosuppression, and obesity predispose unvaccinated patients to delta variant infection. Other predisposing conditions for delta variant infection are sex (male), age, severe asthma, cancer, and pregnancy. Reference [18] reports that patient infected with delta variants are somewhat younger than patients infected with the alpha variant. Furthermore, there is a higher proportion of Asian patients infected with the delta variant in comparison to the alpha variant group. Such difference in infectivity of delta variant affects the comparison analysis of hospitalization and mortality rate outcome. Further studies are needed to assess death rate and the vaccine status.

\section{VACCINE AND DELTA VARIANT}

It is reported that the delta variant can infect unvaccinated as well as vaccinated individuals [13]. Infected vaccinated individuals can further transmit this infection and can cause vaccine breakthrough infections. There is also evidence of the decreased sensitivity of delta variant to vaccine stimulated antibodies and convalescent sera. Soon after the emergence of the delta/B.1.617.2 variant in India in early 2021, vaccine breakthrough infection with the delta variant among vaccinated healthcare workers were observed that led to further transmission chains. Such infections were reported in 3 health care facilities with 3800, 4000 and 1100 healthcare staff members, respectively. In all these facilities, majority of the vaccine-breakthrough infections were caused by delta variant and demonstrated median cycle threshold values under 20 with notable respiratory viral load among fully vaccinated healthcare members. Furthermore, the transmission of delta variant among vaccinated individuals is higher as compared to those infected with B.1.617.1 or B.1.1.7 lineages. The sensitivity of the delta variant to neutralizing immune antibodies elicited by BNT162b2 and ChAdOx-1 vaccines or by convalescent sera demonstrates 8fold reduced sensitivity to both vaccines and sera as compared to that of Wuhan-1 wild type and B.1.1.7 lineage. These findings are also in accordance with the UK data that shows reduced vaccine efficacy against delta variant among fully vaccinated individuals [13].

Although delta variant can infect vaccinated individuals, other studies show decrease in COVID-19 severity among vaccinated individuals specifically after two vaccine doses. A study in Ontario, Canada, analyzed the effectiveness of BNT162b2 (Pfizer-bioNTech), mRNA-1273 (Moderna) and ChAdOx1 (AstraZeneca) vaccines against symptomatic 
COVID-19 infection, hospitalization and death in individuals infected with VOCs. They found the effectiveness of these vaccines against symptomatic delta variant infection $\geq 7$ days after 2 vaccine doses to be $87-95 \%$, that reduced after $\geq 14$ days of $1^{\text {st }}$ dose. mRNA-1273 (70\%) and ChAdOx1 (68\%) vaccines' effectiveness is found to be highest after the $1 \mathrm{st}$ dose. Moreover, the effectiveness of these vaccines is found to be higher against hospitalization or death as compared to that against symptomatic COVID-19 infection after one dose [8]. Similar results were found by a study in Scotland for BNT162b2 and for ChAdOx1 vaccines [19]. A study in the UK analyzing the effectiveness of BNT162b2 and ChAdO1 vaccines against symptomatic COVID-19 disease by testnegative case control study found about $36 \%$ and $30 \%$ vaccine effectiveness respectively, after 1 st vaccine doses and about $88 \%$ and $67 \%$ vaccine effectiveness respectively, after two vaccine doses [11].

Another study in Singapore analyzed the effectiveness of a mRNA licensed vaccine in hospitalized individuals infected with delta variant and found high effectivity of mRNA vaccines against symptomatic and severe COVID-19 infection with the delta variant. Among vaccinated individuals, viral RNA load declines rapidly and a robust antibody response is observed [7]. This demonstrates the effectiveness of all available vaccines in protecting against severe COVID-19 caused by the delta variant.

\section{CONCLUSION}

In conclusion, the high transmission rate, high viral load and the ability to evade immune system make delta variant another VOC of SARS-CoV-2 that if left uncontrolled can cause more devastating effects as compared to that caused by other VOCs. Delta variant of SARS-CoV-2 is capable of causing disease in both vaccinated and unvaccinated individuals. Although complete vaccination does not guarantee $100 \%$ prevention from infection with the various VOCs of SARS-CoV-2, but it provides protection against severe disease. The mRNA-based vaccines are the most effective in this case. Large scale vaccination of individuals should be encouraged to lower disease severity due to infection by different lineages of SARS-CoV-2.

\section{REFERENCES}

[1] Nyberg T, Twohig KA, Harris RJ, Seaman SR, Flannagan J, Allen H, et al. Risk of hospital admission for patients with SARS-CoV-2 variant B.1.1.7: Cohort analysis. The BMJ. 2021; 373: 1-10.

[2] Fisman DN, Tuite AR. Progressive Increase in Virulence of Novel SARS-CoV-2 Variants in Ontario, Canada. MedRxiv, 2021.

[3] O'Toole Á, Hill V, Pybus OG, Watts A, Bogoch II, Khan K, et al. Tracking the international spread of SARS-CoV-2 lineages B. 1.1. 7 and B. 1.351/501Y-V2. Wellcome Open Research. 2021; 6.

[4] World Health Organization. WHO Coronavirus (COVID-19) Dashbooard. [Internet] 2021. [cited on November 28, 2021] Available from: https://covid19.who.int.

[5] Li B, Deng A, Li K, Hu Y, Li Z, Xiong Q, et al. Viral infection and transmission in a large, well-traced outbreak caused by the SARSCoV-2 Delta variant. MedRxiv, 2021.

[6] Shitrit P, Zuckerman NS, Mor O, Gottesman BS, Chowers M. Nosocomial outbreak caused by the SARS-CoV-2 Delta variant in a highly vaccinated population, Israel, July 2021. Eurosurveillance. 2021; 26(39).

[7] Chia PY, Ong SWX, Chiew CJ, Ang LW, Chavatte J-M, Mak T-M., et al. Virological and serological kinetics of SARS-CoV-2 Delta variant vaccine-breakthrough infections: a multi-center cohort study. Clinical Microbiology and Infection. 2021.

[8] Nasreen S, Chung H, He S, Brown KA, Gubbay JB, Buchan SA, et al. Effectiveness of mRNA and ChAdOx1 COVID-19 vaccines against symptomatic SARS-CoV-2 infection and severe outcomes with variants of concern in Ontario. MedRxiv, 2021.

[9] Tuite AR, Fisman DN, Odutayo A, Bobos P, Allen V, Bogoch II, et al. COVID - 19 Hospitalizations, ICU Admissions and Deaths Associated with the New Variants of Concern. Science Briefs of the Ontario COVID-19 Science Advisory Table. 2021; 1(18): 1-10.

[10] Cherian S, Potdar V, Jadhav S, Yadav P, Gupta N, Das M, et al. The Second Wave of COVID-19 in Maharashtra , India. Microorganism. 2021; 2 : $1-11$.

[11] Lopez Bernal J, Andrews N, Gower C, Gallagher E, Simmons R, Thelwall S, et al. Effectiveness of Covid-19 Vaccines against the B.1.617.2 (Delta) Variant. New England Journal of Medicine. 2021; 385(7): 585-594.

[12] Centers for Disease Control and Prevention. COVID Data Tracker. [Internet] 2021. [cited on November 29, 2021] Available from: https://covid.cdc.gov/covid-data-tracker

[13] Mlcochova P, Kemp S, Dhar MS, Papa G, Meng B, Ferreira IATM, et al. SARS-CoV-2 B.1.617.2 Delta variant replication and immune evasion. Nature. 2021; 599(November).

[14] Kuppalli K, Gala P, Cherabuddi K, Kalantri SP, Mohanan M, Mukherjee B, et al. India's COVID-19 crisis: a call for international action. The Lancet. 2021; 397(10290): 2132-2135.

[15] Mishra S, Mindermann S, Sharma M, Whittaker C, Mellan TA, Wilton $\mathrm{T}$, et al. Changing composition of SARS-CoV-2 lineages and rise of Delta variant in England. EClinicalMedicine. 2021; 39: 1-8.

[16] Organization PAH. (2021, October 14). Epidemiological update: Coronavirus disease (covid-19) [Internet] 2014. [cited on November 24, 2021] Available from: https://iris.paho.org/handle/10665.2/54997.

[17] Gallo Marin B, Aghagoli G, Lavine K, Yang L, Siff EJ, Chiang SS, et al. Predictors of COVID-19 severity: A literature review. Reviews in Medical Virology. 2021; 31(1): 1-10.

[18] Twohig KA, Nyberg T, Zaidi A, Thelwall S, Sinnathamby MA, Aliabadi S, et al. Hospital admission and emergency care attendance risk for SARS-CoV-2 delta (B.1.617.2) compared with alpha (B.1.1.7) variants of concern: a cohort study. Lancet Infect Dis. 2021.

[19] Sheikh A, McMenamin J, Taylor B, Robertson, C. SARS-CoV-2 Delta VOC in Scotland: demographics, risk of hospital admission, and vaccine effectiveness. Lancet (London, England). 2021; 397(10293): 2461-2462. 